\title{
Adverse childhood experiences and depressive symptoms in later life: Longitudinal mediation effects of inflammation
}

\author{
${\text { Eleonora } \text { Iob }^{\mathrm{a}, *} \text {, Rebecca Lacey }}^{\mathrm{b}}$, Andrew Steptoe ${ }^{\mathrm{a}}$

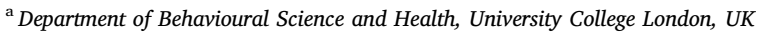 \\ ${ }^{\mathrm{b}}$ Department of Epidemiology and Public Health, University College London, UK
}

\section{A R T I C L E I N F O}

\section{Keywords:}

Adverse childhood experiences

C-reactive protein

Depressive symptoms

Longitudinal mediation

Older adults

\begin{abstract}
A B S T R A C T
Background: Adverse childhood experiences (ACEs) have been associated with both inflammation and depression. However, few studies have examined the role of inflammation as a possible biological mechanism underlying the association of ACEs with depression in later life using longitudinal data. This study investigated the longitudinal mediation effects of inflammation in the relationship between ACEs and depressive symptoms in older adults.

Methods: We utilised data from the English Longitudinal Study of Ageing ( $\mathrm{N}=4382)$. ACEs (i.e. threat, family dysfunction, low parental bonding, loss experiences) were assessed retrospectively at wave 3 (2006/07). Creactive protein (CRP), an inflammatory marker, was measured at waves 2 (2004/05), 4 (2008/09), and 6 (2012/13). Depressive symptoms were ascertained from wave 6 to $8(2016 / 17)$. The mediation analysis was conducted using parallel process latent growth curve modelling.

Results: Greater ACEs cumulative exposure was associated with higher CRP and depressive symptoms at baseline $\left(\beta_{\mathrm{CRPi}}=0.066[0.030-0.102] ; \quad \beta_{\mathrm{DEPi}}=0.149[0.115-0.183]\right)$ and with their increase over time $\left(\beta_{\mathrm{CRPs}}=0.205[0.095-0.315] ; \beta_{\mathrm{DEPs}}=0.355[0.184-0.526]\right)$. Baseline CRP levels were positively associated with baseline depressive symptoms ( $\left.\beta_{\mathrm{DEPi}}=0.145[0.104-0.186]\right)$ and their trajectory $\left(\beta_{\text {DEPs }}=0.215[0.124-0.306]\right)$. The mediation analysis indicated that higher baseline CRP levels mediated respectively $7 \%$ and $5 \%$ of the total effect of ACEs cumulative exposure on the baseline value and change in depressive symptoms. These mediation effects were larger for Loss experiences (i.e. $20 \%$ and $12 \%$ respectively) than for other types of ACEs. In addition, they were independent of possible confounders and additional mediators including adult socioeconomic position and lifestyle factors.

Conclusion: ACEs were related to higher depressive symptoms partly via elevated CRP levels. Inflammation might be one of the psychobiological mechanisms underlying the link between ACEs and depression. Psychosocial and behavioural interventions to prevent and reduce the negative impact of ACEs might help to lower the risk of inflammation and depression in the population.
\end{abstract}

\section{Introduction}

Depression is the predominant mental disorder across the world affecting $>300$ million people globally (Patel, 2016). It is amongst the leading causes of the global burden of disease and disability (James, 2018). Additionally, it is associated with the development of other physical diseases and premature mortality (Kivimäki et al., 2019). The burden of depression is higher amongst older adults compared with younger people (Global Burden of Disease Collaborative Network, 2018), possibly due to increasing cognitive and physical impairments and diminishing social connections (Kok and Reynolds, 2017). For instance, $22 \%$ of men and $28 \%$ of women aged over 65 years are estimated to experience significant depressive symptoms in the UK (College, 2018). Cross-sectional and longitudinal studies suggest that depressive symptoms tend to exhibit a U-shape trajectory across the life course, characterised by relatively high levels of depressive symptoms in early adulthood, lower symptoms during middle age, and elevated symptoms again during later life (Tampubolon and Maharani, 2017). On the other hand, there is some evidence suggesting that older age could also represent a protective factor for depression due to better emotion regulation, positive reappraisal of negative events, higher selfesteem, and greater meaningful engagement in social activities,

\footnotetext{
* Corresponding author at: Research Department of Behavioural Science and Health, Institute of Epidemiology and Health Care, University College London, 1-19 Torrington Place, London WC1E 7HB, UK.

E-mail address: eleonora.iob.17@ucl.ac.uk (E. Iob).
} 
religion, or volunteering (Fiske et al., 2009). Understanding the biological, psychological, and social factors that may increase the risk of depression among older adults, or offer protection against its development, is an important public health priority owing to the ongoing increase in the pace of population ageing in numerous countries (Rodda et al., 2011).

Adverse childhood experiences (ACEs) can be defined as those earlylife experiences that are likely to require considerable psychological, social or neurobiological adaptation by an average child and which represent a deviation from the expectable environment (McLaughlin, 2016). The first major investigations into the effects of ACEs on health and child development considered adversities such as abuse (emotional, physical, and sexual), neglect (emotional and physical), and experiences of household dysfunction (e.g. violence between parents, parental separation, and household members affected by substance misuse, mental illness or criminal behaviour) (Felitti, 1998; Dong, 2004). More recently, studies on ACEs have also incorporated additional adversities to represent other important domains of childhood experiences that might be important in predicting long-term health and well-being outcomes (Finkelhor et al., 2015, 2013), such as parent-child relationships, bullying, and neighbourhood violence (Hughes, 2017; Houtepen et al., 2018). ACEs are extremely common across several countries (Bellis et al., 2014; Merrick et al., 2018), and are major psychosocial risk factors for depression, as well as for other mental and physical disorders (Hughes, 2017; Bellis, 2019). Overall, individuals who experienced four or more ACEs are three times more likely to develop depression compared with those without ACEs (Hughes, 2017). ACEs have been associated not only with the development of depression, but also with worse prognosis and treatment outcomes amongst depressed indviduals (Nanni et al., 2012). Relatively little is known about the role of ACEs in late-life depression since most studies have utilised samples of mixed ages excluding the oldest age groups. Nevertheless, recent evidence suggests that the adverse effects of earlylife stress on mental health are likely to persist into later life (Cheong et al., 2017).

Maladaptive alterations in the function of the inflammatory response system could underlie the long-term biological impact of ACEs on depression. Observational human studies and experimental animal models suggest that exposure to severe and chronic stress during early life might adversely affect the development of the immune system, leading to chronically elevated and hyperactive inflammatory responses. This in turn may impair the development and function of the brain thereby increasing the individual's vulnerability to stress-related psychopathologies such as depression (Danese and Baldwin, 2017). Numerous studies have found that individuals exposed to multiple ACEs have increased plasma levels of inflammatory markers such as C-reactive protein (CRP) several decades later. A meta-analysis of 25 studies confirmed the relationship between different types of ACEs and elevated inflammation in adults (mean age $=42$ years) (Baumeister et al., 2016). Furthermore, a recent investigation has demonstrated that ACEs were associated with high CRP levels in a large sample of older adults (mean age $=70$ years) (Iob et al., 2019). On the other hand, increased levels of inflammation have been implicated in the pathogenesis of depression (Miller et al., 2009). Meta-analyses of the literature have indicated that depressed individuals tend to have elevated concentrations of various inflammatory markers, both peripherally and in the brain (Haapakoski et al., 2015; Enache et al., 2019).

A large number of studies have investigated the relationship of inflammation with either ACEs or depression. Yet, most research has not formally tested the role of inflammation as a mediating mechanism through which ACEs may become biologically embedded and increase the risk of depression. One study found that greater exposure to psychosocial stressors was related to the onset of depressive symptoms partly via increasing levels of interleukins (i.e. inflammatory marker) in a sample of adolescents (Flouri et al., 2020). However, there are virtually no studies focusing on older adults. Importantly, the contribution of inflammation to depression might be particularly relevant to older adults. Inflammatory responses in healthy adults have been shown to increase in an age-dependent fashion (Tang et al., 2017). In addition, elevated inflammation is linked to various long-term conditions such as cardiovascular disease and diabetes which tend to emerge at older ages. New evidence also indicates that the relationship of ACEs with inflammatory markers might be stronger in adults compared with children and adolescents (Kuhlman et al., 2019). Hence, a better understanding of how elevated inflammation in the context of ACEs might influence the risk of depression amongst older adults will be instrumental in developing more targeted intervention and prevention strategies. Another important evidence gap concerns the prevailing use of cumulative risk scores of ACEs which ignore the potentially different physiological consequences of different ACEs dimensions (McLaughlin and Sheridan, 2016). For instance, preliminary evidence suggests that ACEs involving harm or threat of harm might have more powerful effects on stress-related biological and cognitive outcomes than ACEs related to deprivation (i.e. absence of expected cognitive and social input) (Busso et al., 2017; Sumner et al., 2019). In addition, one study employing latent class analysis demonstrated that ACEs clusters including experiences of household dysfunction, parental loss, and maltreatment were particularly important for elevated inflammation in mid-life (Lacey et al., 2020). Lastly, most studies have employed crosssectional measures of inflammation and depressive symptoms which do not permit to consider the temporal dynamics of their relationship.

This study aimed to address these evidence gaps by testing the mediation effects of trajectories of CRP (i.e. inflammatory marker) in the relationship between different dimensions of ACEs and depressive symptom trajectories in a large sample of older adults. First, we expected that greater ACEs exposure would be associated with elevated CRP and depressive symptoms at baseline and predict an increase in their trajectories over time. Second, we hypothesised that the associations of ACEs with depressive symptoms would be mediated by elevated CRP levels. Third, we expected that distinct ACEs dimensions would exhibit differential relationships with inflammation and depressive symptoms.

\section{Materials and methods}

\subsection{Sample}

The data came from the English Longitudinal Study of Ageing (ELSA), an ongoing prospective population-based cohort study of older adults aged 50 years and over living in England (Zaninotto and Steptoe, 2019). The study began in 2002 with an original sample of 12,099 participants, who were drawn from the Health Survey for England. This sample was representative of the general English population of older adults (Steptoe et al., 2013), and was not selected on the basis of exposure to ACEs or presence of depressive symptoms. Further details about the sample design and methods of data collection can be found on the study website (www.elsa-project.ac.uk). Retrospective data on ACEs were collected during the Life History Interview in wave $3(2006 / 07)$. Plasma concentrations of CRP were measured in waves 2, 4, and 6 (2004/05-2012/13). Depressive symptoms were assessed from wave 6 $(2012 / 13)$ to wave $8(2016 / 17)$ in order to represent the temporal order of their relationship with inflammation (Fig. 1). The analytical sample included all participants who completed the Life History Interview at wave $3(\mathrm{~N}=7855)$, who also had available measures of CRP and depressive symptoms on at least one occasion $(\mathrm{N}=5627)$. In addition, study members with CRP values $>10 \mathrm{mg} / \mathrm{L}$ were excluded from the study $\left(\mathrm{N}_{\mathrm{wave} 2}=459 ; \mathrm{N}_{\text {wave } 4}=444 ; \mathrm{N}_{\text {wave6 }}=342\right)$ since this could reflect current infection or trauma rather than chronic inflammation (Pearson, 2003). This resulted in a final analytical sample of 4382 participants. All respondents provided informed consent, and ethical approval was obtained from the National Research Ethics Service. 


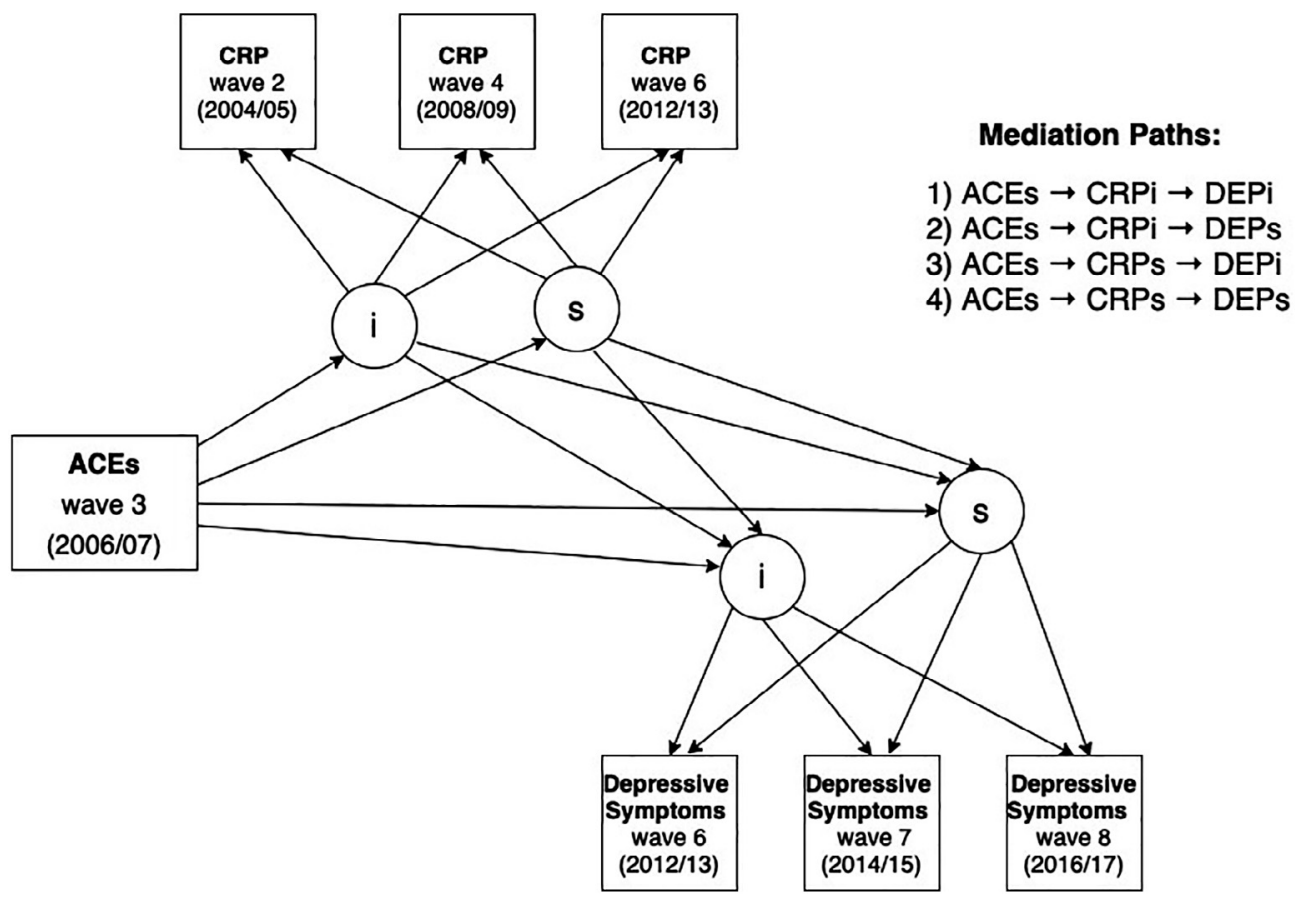

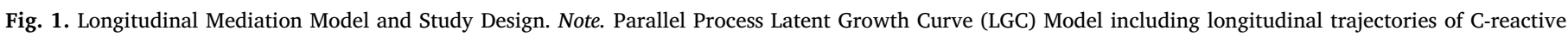
protein (CRP) and depressive symptoms with four mediation paths. ACEs = Adverse Childhood Experiences; $\mathrm{i}=\mathrm{Intercept;} \mathrm{s}=\mathrm{Slope}$.

\subsection{Measures}

\subsubsection{Exposures}

The Life History interview gathered information about different types of ACEs experienced up to the age of 16 years. These include: sexual abuse, physical assault, physical abuse from parents, parent arguments, parent mental illness or substance abuse, parent separation or divorce, maternal bonding, paternal bonding, separation from mother for more than six months, parent death, foster care or adoption, and institutionalisation (Table 1). The selection of these ACE items was informed by the ACEs definition presented in the Introduction (McLaughlin, 2016), and by recent studies in this field which have considered a wider range of adversities compared with the first major ACE investigations to provide a more comprehensive assessment of this construct. For all items, except parental bonding, participants reported whether or not they ever experienced that particular event during childhood. Child-parent relationships were assessed using the sevenitem Parental Bonding Instrument (PBI) (Parker et al., 1979). Low maternal and paternal bonding were defined as total PBI scores $\geq 3$, since the majority of participants (around $80 \%$ ) had a score lower than 3 . Based on these items, we calculated a cumulative risk score representing the total number of ACEs experienced by the participant. In addition, we examined the effects of different ACEs dimensions which we identified in our previous dimensional analysis of ACEs in ELSA using explorative and confirmatory factor analysis with cross-validation (Iob et al., 2019). These were: Threat (sexual abuse, physical assault, physical abuse from parents), Household Dysfunction (parent arguments, parent mental illness or substance abuse, parent separation or divorce), Low Parental Bonding (poor maternal and paternal bonding), and Loss experiences (separation from mother for more than six months, parent death, foster care or adoption, and institutionalisation). Each dimension was measured using a dichotomous score representing the presence or absence of at least one type of ACE relevant to that dimension. It is worth noting that we did not include childhood socioeconomic disadvantage in our ACEs measures since it is conceptually different from the types of psychosocial adversities considered in this study. Childhood socioeconomic factors represent the financial, material, and sociocultural circumstances in which children grow up and might affect mental health through different mechanisms (TaylorRobinson et al., 2018; Amso and Lynn, 2017). In addition, numerous studies have suggested that childhood socioeconomic disadvantage is an important risk factor for ACEs (Walsh et al., 2019). We therefore included childhood socioeconomic position as a covariate in the analysis. Further details about the specific questions included in the Life History Interview can be found in the User Guide (Ward et al., 2009).

\subsubsection{Mediator}

Blood samples for the assessment of CRP were collected during the nurse visits. All participants who gave consent were eligible for a blood sample to be taken. The only exclusion criteria for blood sampling were: clotting or bleeding disorders, history of fits or convulsions, and being on anticoagulant medication. Participants were asked to fast before their nurse visit unless they had diabetes and were on treatment or if they were unfit to fast. A minimum of three small tubes of blood (ranging in size from $2 \mathrm{ml}$ to $6 \mathrm{ml}$ ) were collected from each respondent. After collection, the tubes were sent to the Department of Clinical Biochemistry at the Royal Victoria Infirmary in Newcastle (UK), and were then frozen for long-term storage. Plasma concentrations of CRP were assayed in duplicate from the $6 \mathrm{ml}$ plain tubes using the N Latex CRP mono Immunoassay on the Behring Nephelometer II Analyser (Dade Behring, Milton Keynes, UK). The CRP values were expressed in $\mathrm{mg} / \mathrm{L}$. The coefficients of variation ranged between $3 \%$ and $7 \%$. The reference range for CRP used by the laboratory was $0-5 \mathrm{mg} / \mathrm{L}$. Values within this range were considered to be clinically 'normal', while those outside were treated as clinically 'abnormal'. For the present analysis, the CRP measures were log-transformed since their distribution was positively skewed. Further details about the blood sample analyses, internal quality control, and external quality assessment of the laboratory can be found in the ELSA Nurse Visit documentation (NatCen Social Research, 2018) and the 2004 Health Survey for England technical report (Graig et al., 2004), which employed the same laboratory, guidelines, and procedures for the analysis of blood biomarkers as those used in ELSA. 
Table 1

Descriptive statistics and comparison of observed and imputed data.

\begin{tabular}{|c|c|c|c|c|}
\hline Variable & Levels & $\begin{array}{l}\text { Observed } \\
\% \text { (Counts) }\end{array}$ & $\begin{array}{l}\text { Missing } \\
\% \text { (Counts) }\end{array}$ & $\begin{array}{l}\text { Imputed }^{\mathrm{a}} \\
\%\end{array}$ \\
\hline \multicolumn{5}{|l|}{ Adverse childhood experiences (ACEs) } \\
\hline Physical abuse & & $3.2\left(\begin{array}{lll}1 & 1 & 9\end{array}\right)$ & $14.9(653)$ & 3.4 \\
\hline Sexual abuse & & $5.2\left(\begin{array}{lll}1 & 9 & 4\end{array}\right)$ & $15.1(660)$ & 5.4 \\
\hline Physical assault & & $2.7(99)$ & $15.0(656)$ & 2.8 \\
\hline Parent arguments & & $20.4(751)$ & $15.8(694)$ & 20.9 \\
\hline Low maternal bonding & & $17(598)$ & $19.7(863)$ & 17.3 \\
\hline Low paternal bonding & & $17.3(591)$ & $22.1(967)$ & 18.4 \\
\hline Institutionalisation & & $1.6(70)$ & $0.1(3)$ & 1.6 \\
\hline Separation from mother & & $14.6(639)$ & $0.1(3)$ & 14.6 \\
\hline Foster care/Adoption & & $1.8(78)$ & $0.1(3)$ & 1.8 \\
\hline Parent death & & $5.2(226)$ & $0.2(7)$ & 5.2 \\
\hline Parent mental illness/substance abuse & & $6.3(235)$ & $15.2(668)$ & 6.5 \\
\hline Parent separation/divorce & & $5.7(251)$ & $0.2(7)$ & 5.8 \\
\hline \multicolumn{5}{|l|}{ CRP (mg/L) - Mean (sd) } \\
\hline w2 & & $2.42(2.12)$ & $32(1405)$ & $2.48(2.17)$ \\
\hline w4 & & $2.41(2.13)$ & $28(1241)$ & $2.56(2.22)$ \\
\hline w6 & & $2.19(1.98)$ & $24(1033)$ & $2.35(2.01)$ \\
\hline \multicolumn{5}{|c|}{ CESD-8 (total score) - Mean (sd) $/ \% \geq 3$ symptoms } \\
\hline w6 & & $1.24(1.79) / 18.2$ & $0.1(38)$ & $1.25(1.80) / 18.4$ \\
\hline w7 & & $1.28(1.75) / 17.5$ & $12.2(533)$ & $1.36(1.82) / 19.2$ \\
\hline w8 & & $1.27(1.72) / 18.1$ & $21.7(951)$ & $1.44(1.86) / 21.6$ \\
\hline \multicolumn{5}{|l|}{ Demographic and socioeconomic characteristics } \\
\hline Age & & $69.94(8.82)$ & 0.0 & $69.94(8.82)$ \\
\hline Sex: Female & & $56(2453)$ & 0.0 & 56 \\
\hline \multirow[t]{4}{*}{ Marital status } & Married & $65.2(2856)$ & $0.1(4)$ & 65.2 \\
\hline & Separated/Divorced & $11(483)$ & & 11 \\
\hline & Windowed & $18.3\left(\begin{array}{lll}8 & 0 & 3\end{array}\right)$ & & 18.3 \\
\hline & Single & $5.5(239)$ & & 5.5 \\
\hline \multirow[t]{5}{*}{ Wealth quintiles } & 1 (lowest) & $15.4(664)$ & $1.8(79)$ & 15.4 \\
\hline & 2 & $18.0(775)$ & & 18.0 \\
\hline & 3 & $20.4(878)$ & & 20.4 \\
\hline & 4 & $22.4(963)$ & & 22.4 \\
\hline & 5 (highest) & $23.8(1023)$ & & 23.8 \\
\hline \multicolumn{5}{|l|}{ Childhood socioeconomic position } \\
\hline Overcrowding & & $19.4(819)$ & $3.9(170)$ & 19.5 \\
\hline No books when aged 10 & & $25(1045)$ & $4.5(196)$ & 25.1 \\
\hline Manual occupation (father) & & $1.1(48)$ & $0.2(7)$ & 1.1 \\
\hline Financial hardship & & $6.8(253)$ & $15.3(669)$ & 7.0 \\
\hline Parent unemployment & & $8(298)$ & $15.3(671)$ & 8.3 \\
\hline \multicolumn{5}{|l|}{ Lifestyle indicators } \\
\hline BMI - Mean (sd) & & $28.2(5.1)$ & $4.9(219)$ & $28.3(5.1)$ \\
\hline Smoking & Current smoker & $16.1(703)$ & $0.2(8)$ & 16.1 \\
\hline \multirow[t]{3}{*}{ Physical activity } & Light & $15.5(672)$ & $1.1(47)$ & 15.5 \\
\hline & Moderate & $51.0(2209)$ & & 51.0 \\
\hline & Vigorous & $33.5(1454)$ & & 33.5 \\
\hline \multirow{3}{*}{ Alcohol consumption } & 5-7 days a week & $24.3(952)$ & $10.7(467)$ & 23.9 \\
\hline & 1-4 days a week & $39.6(1551)$ & & 39.4 \\
\hline & Less than weekly & $36.1(1412)$ & & 36.7 \\
\hline \multicolumn{5}{|l|}{ Medications } \\
\hline Anti-inflammatory /antihypertensive drugs & Yes & 48.8 (2137) & 0.0 & 48.8 \\
\hline
\end{tabular}

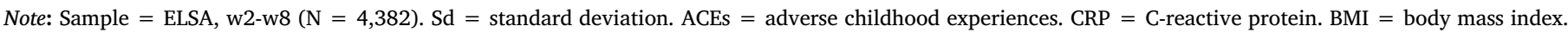
CESD-8 = 8-item Centre for Epidemiological Studies-Depression scale.

${ }^{\mathrm{a}}$ Only percentages are given for the imputed data since frequencies vary across the 20 imputed datasets.

\subsubsection{Outcome}

Depressive symptoms were ascertained using the 8-item Centre for Epidemiological Studies Depression scale (CESD-8), which measures eight different cognitive-affective (i.e. "enjoyed life", "felt depressed", "happy", "lonely", and "felt sad") and somatic symptoms of depression ("everything I did was an effort", "sleep was restless", and "I could not get going") (Radloff, 1977). A dichotomous (yes/no) response was used for each item, resulting in a total CESD-8 score ranging between 0 (no symptoms) and 8 (all eight symptoms). This short version of the CESD has been widely used in studies of late life depression (White, 2016; Zivin, 2010), and has good psychometric properties for use in these populations (Karim et al., 2015; Andresen et al., 1994). It has also been shown to have good internal consistency at each wave of ELSA
(Cronbach's a > 0.95) (White, 2016), and comparable psychometric properties to the full 20-item CESD (Radloff, 1977; Turvey et al., 1999). A cut-off of 3 or more symptoms is typically used to identify cases of depression or significant depressive symptoms. This definition was validated against standardised psychiatric interviews in older populations (Turvey et al., 1999). At each wave, we calculated the total CESD8 score to assess the overall number of depressive symptoms reported by the participant.

\subsubsection{Covariates}

We included a number of covariates which were selected based on previous studies in the field and through the use of directed acyclic graphs (DAGs; Supplement, eFig. 1). Sex, age, and childhood 

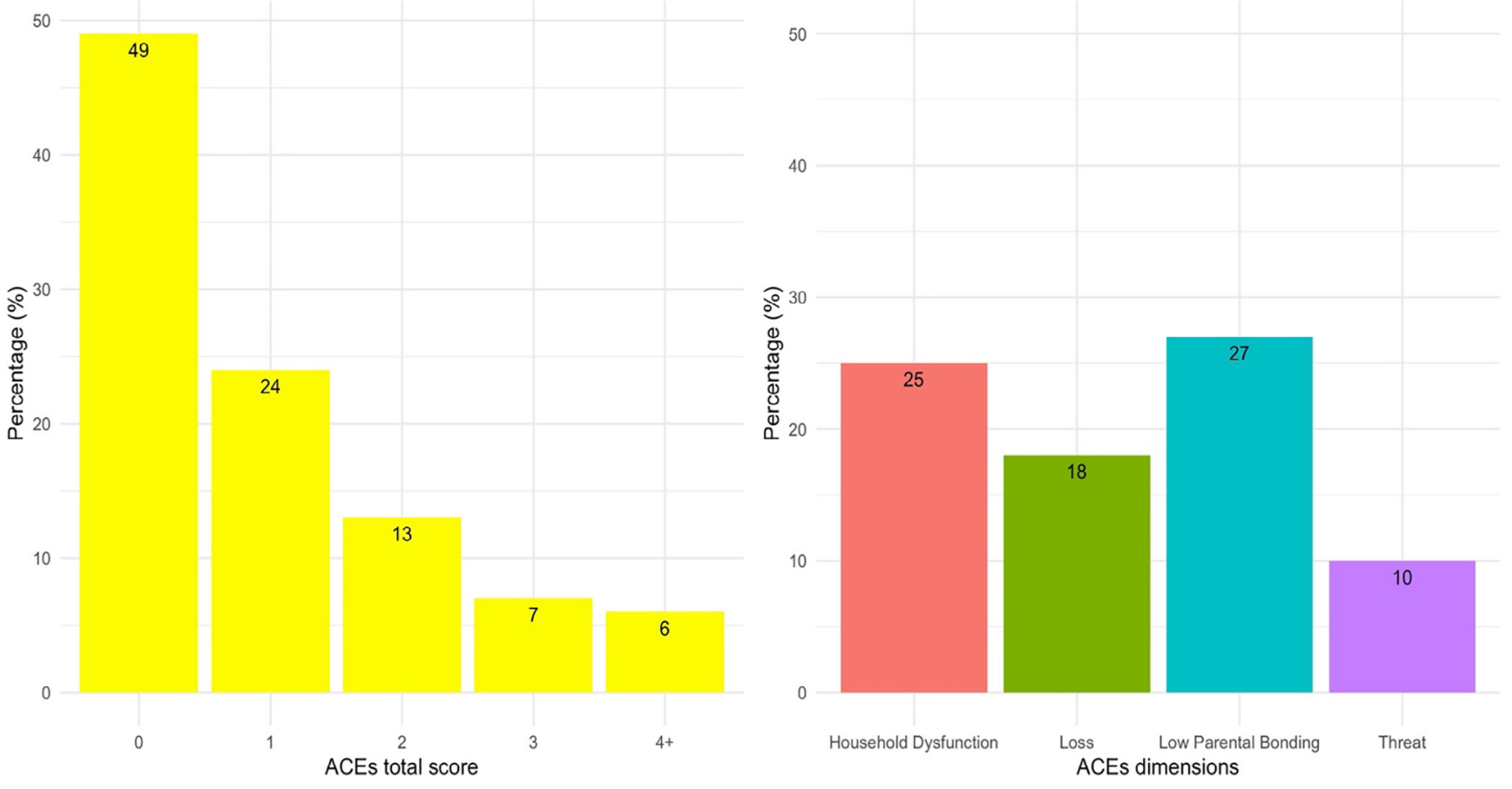

Fig. 2. Prevalence of ACEs in the ELSA sample. Note. ACEs total score and dimensions (i.e. Household Dysfunction, Loss, Low Parental Bonding, and Threat).

socioeconomic position (overcrowding, number of books in the home, father's occupation, financial hardship, and parent unemployment) were included as possible confounders in all models. The analyses were also adjusted for the use of anti-inflammatory or antihypertensive drugs which may alter CRP levels. As indicated by our DAG, lifestyle factors [i.e. body mass index (BMI), smoking status, physical activity, and alcohol drinking] and adult socioeconomic position were intermediate variables on the causal pathways linking ACEs with depressive symptoms. Hence, they were included in the models as additional mediators rather than confounders in order to avoid overadjustment bias (Schisterman et al., 2009). The inclusion of these covariates enabled us to measure the extent to which CRP explained the association between ACEs and depressive symptoms after accounting for possible confounders and additional mediators. A more detailed description of the measurement and coding of the covariates can be found in the Supplement (eMethods).

\subsection{Statistical analyses}

The longitudinal mediation analysis was conducted using parallel process Latent Growth Curve (LGC) modelling (Selig and Preacher, 2009). This technique allows to estimate a mediation model including longitudinal trajectories of both the mediator and the outcome simultaneously (Fig. 1). The mediation effects of CRP were tested by calculating the indirect association of ACEs with depressive symptoms through CRP after other covariates had been taken into account using the 'Product of Coefficients' method (MacKinnon et al., 2007). The parallel process LGC mediation model includes four types of mediation effects since the intercept (i) and slope (s) of the growth trajectories are both part of the mediation paths (see Fig. 1). All direct and indirect associations were calculated using bias-corrected bootstrapped standard errors based on 5,000 samples (Hayes, 2009). First, we fit an unconditional parallel process LGC model of CRP and depressive symptoms without risk factors. Second, we introduced the ACEs total score/dimension and all covariates (confounders and additional mediators) in the model to test the mediation paths illustrated in Fig. 1 (Model 1; see example of model syntax in the Supplement, eMethods). We tested one LGC mediation model for the ACEs cumulative score and one for each ACEs dimension. All models were estimated using robust weighted least squares estimation since depressive symptoms were treated as ordinal variables. As a sensitivity analysis, we tested the direct and indirect associations between ACEs, CRP, and depressive symptoms without adjustment for additional mediators (i.e. adult lifestyle factors and socioeconomic position; Model 2). In addition, we calculated E-values and least extreme confidence limits for all statistically significant direct effects in order to determine the minimum strength of the association on the risk ratio scale that an unmeasured confounder should have with both the exposure and the outcome to fully explain their relationhsip (VanderWeele and Ding, 2017). Missing data on the exposure, mediator, outcome, and covariate variables were estimated using multiple imputation by chained equations (MI). All variables included in the analysis were used as predictors in the imputation model in addition to additional data on physical health, survey weights, and covariates from other waves of the study. We created twenty imputed datasets, and the estimates from the LGC mediation analysis were pooled used Rubin's rules (Rubin, 2004). Data management and MI analysis were performed in Rstudio 3.4.4. The LGC models were estimated in Mplus 7. An overview of the fit indices used to assess the adequacy of the models can be found in the Supplement (eMethods).

\section{Results}

\subsection{Descriptive statistics}

The characteristics of the study participants in the observed and imputed data are reported in Table 1 . The average age was 70 years ( $56 \%$ female). There was a higher proportion of participants in the highest compared with the lowest wealth quintiles. Depressive symptoms were generally low (mean total CESD-8 score $=1.3$ ) but ranged across the full spectrum from zero to eight. Around $18 \%$ of the participants had elevated depressive symptoms ( $\geq 3$ symptoms), which is lower than the estimated prevalence of depression among older people in the UK (22\% in men and $28 \%$ in women) (College, 2018). The mean CRP value at baseline was $1.63 \mathrm{mg} / \mathrm{L}$. Around $50 \%$ of the sample did not have any ACEs, whereas $7 \%$ reported 3 ACEs, and another $6 \%$ of participants had 4 or more ACEs (Fig. 2). The percentage of participants who reported at least one ACE related to Household Dysfunction, Loss, 
Low Parental Bonding, or Threat was respectively 25\%, 18\%, 27\%, and $10 \%$ (Fig. 2). The observed and imputed values of the participants' characteristics were broadly similar, indicating that the multiple imputation method achieved its goals (Table 1).

\subsection{Unconditional parallel process LGC Model of CRP and depressive symptoms}

The unconditional parallel process LGC model of CRP and depressive symptoms without risk factors fit the data well, RMSEA $=0.027$, $\mathrm{CFI}=0.995$, TLI $=0.990$. The average expected value of log CRP at wave 2 (intercept, i.e. baseline) was 0.53 . The mean of the slope (i.e. change) was $-0.02(\mathrm{p}<.001)$, suggesting that CRP decreased by 0.02 points in each consecutive wave of the study, on average. The estimated mean number of depressive symptoms at wave 6 (intercept; i.e. baseline) was 1.25 . The mean of the slope (i.e. change) was $0.04(\mathrm{p}=.002)$, indicating that the mean number of depressive symptoms increased by 0.04 points in each consecutive wave.

\subsection{Direct associations between ACEs, CRP, and depressive symptoms}

The direct associations between ACEs, CRP, and depressive symptoms adjusted for possible confounders and additional mediators (Model 1) are reported in Table 2 and illustrated in Fig. 3. The ACEs total score was positively associated with the intercept and slope of both CRP and depressive symptoms $\left(\beta_{\mathrm{CRPi}}=0.066[0.030-0.102]\right.$; $\beta_{\mathrm{CRPs}}=0.205[0.095-0.315] ; \quad \beta_{\mathrm{DEPi}}=0.149[0.115,0.183]$; $\beta_{\text {DEPs }}=0.355[0.184-0.526]$. Although the average CRP score in the sample decreased during the study, the positive effect of ACEs on the CRP slope indicates that CRP levels increased amongst participants with a higher number of ACEs. The intercept of CRP was positively associated with both the intercept and slope of depressive symptoms $\left(\beta_{\mathrm{DEPi}}=0.145[0.104-0.186] ; \beta_{\mathrm{DEPs}}=0.215[0.124-0.306]\right)$ independently of ACEs and other covariates. In addition, there was a positive association between the slope of CRP and the intercept of depressive symptoms $\left(\beta_{\mathrm{DEPi}}=0.339[0.037-0.641]\right)$.

In relation to the direct associations of the ACEs dimensions, Loss was positively associated with both the intercept and slope of CRP $\left(\beta_{\mathrm{CRPi}}=0.049[0.017-0.081] ; \beta_{\mathrm{CRPs}}=0.155[0.054-0.256]\right)$, but not with depressive symptoms. Household Dysfunction $\left(\beta_{\text {DEPi }}=0.110[0.073-0.147] ; \beta_{\text {DEPs }}=0.199[0.113-0.285]\right)$ and Low Parental Bonding $\quad\left(\beta_{\mathrm{DEPi}}=0.125[0.088-0.162]\right.$; $\beta_{\text {DEPs }}=0.250[0.168-0.332]$ ) were both positively associated with the intercept and slope of depressive symptoms. In addition, Household Dysfunction was positively associated with the CRP intercept $\left(\beta_{\mathrm{CRPi}}=0.038[0.005-0.071]\right)$, while Low Parental Bonding was positively associated with the CRP slope ( $\left.\beta_{\text {CRPs }}=0.119[0.002-0.236]\right)$. Threat had a positive association only with the slope of depressive symptoms $\left(\beta_{\mathrm{DEPs}}=0.149[0.066-0.232)\right]$. The direct associations of CRP with depressive symptoms in the dimension-specific models were similar to those found in the model with the ACEs total score (Table 2).

\subsection{Indirect associations of ACEs with depressive symptoms through CRP}

The indirect associations of the mediation models adjusted for possible confounders and additional mediators (Model 1) are reported in Table 3. For the ACEs total score, we found a small positive indirect association of ACEs via the CRP intercept with the intercept (ind $=0.006[0.002-0.010]$ ) and slope (ind $=0.003[0.001-0.005]$ ) of depressive symptoms. These results indicate that baseline CRP levels mediated $7 \%$ and $5 \%$ of the association of ACEs with the intercept and slope of depressive symptoms respectively, independently of possible confounders and additional mediators. The indirect associations involving the slope of CRP were almost null and did not reach statistical significance. The mediation effects of baseline CRP levels were larger for Loss experiences than for the other ACEs dimensions. In this case, the intercept of CRP mediated respectively $20 \%$ (ind $=0.016[0.004-0.028]$ ) and $12 \%$ (ind $=0.007[0.001-0.013]$ ) of the association of Loss with the intercept and slope of depressive symptoms after controlling for other study covariates. There also was a positive indirect association of Household Dysfunction with the intercept of depressive symptoms through baseline CRP (ind $=0.011[0.001-0.021]$ ). In contrast, the indirect effects of CRP in the pathways linking Threat and Low Parental Bonding with depressive symptoms were smaller and nonsignificant. Although the indirect associations of Loss experiences were significantly larger than those of the other dimensions, the magnitude of such differences was small across most comparisons (Supplement, eTable 1).

\subsection{Sensitivity analyses}

The pattern of relationships between ACEs, CRP, and depressive symptoms found in the models without additional mediators (i.e. adult lifestyle factors and socioeconomic position) aligned closely to the results of the main analysis including these factors (Model 2, Supplement, eTable2), although some associations were larger when the additional mediators were not taken into account. Likewise, the size of the indirect associations of ACEs with depressive symptoms through CRP was almost unchanged in the models without additional mediators (Model 2, Table 3).

The E-values of the direct associations between ACEs, CRP, and depressive symptoms for the main imputed data analysis (Model 1) ranged between 1.16 (lower limit $=1.05$ ) and 1.66 (lower limit $=1.16)($ Table 2). Although there are no specified ranges of Evalues that are deemed large or small, this suggests that relatively modest confounder associations could explain away the observed effects of ACEs and CRP. Therefore, it is important to consider potential sources of unmeasured confounding when interpreting these findings (Blum et al., 2020) (see Section 4).

The mediation models were reanalysed in the sample of participants with complete data on all variables $(\mathrm{N}=3498)$. The direct and indirect associations between ACEs, CRP, and depressive symptoms were broadly similar to those found in the analyses with the imputed data (Supplement, eTables 3 and 4).

We found significant differences in socioeconomic, health, and lifestyle characteristics between the subsample of ELSA participants included in the study and those excluded owing to insufficient data (i.e. unavailability of at least one measure of ACEs, CRP, and depressive symptoms). However, the magnitude of these differences was small (Supplement, eTable 5).

\section{Discussion}

This is the first prospective study to test the mediation role of CRP in the psychobiological pathways underlying the relationship between ACEs and depression in a large sample of older adults using repeated measures of CRP and depressive symptoms. Our results indicate that greater cumulative exposure to ACEs was associated with elevated CRP levels and depressive symptoms at baseline and with their increase over time. This corroborates the evidence for the long-term association of ACEs with inflammation and depression. Baseline CRP levels were positively associated with depressive symptoms measured 8 years later. Additionally, they predicted an increase in depressive symptoms over the following waves. The mediation analysis indicated that ACEs were associated with depressive symptoms partly through elevated CRP levels. In particular, baseline CRP explained respectively $7 \%$ and $5 \%$ of the association between ACEs cumulative exposure and the baseline value and change in depressive symptoms, independently of possible confounders (sex, age, childhood socioeconomic position) and additional mediators (adult socioeconomic position and lifestyle factors). The mediation effects of CRP were larger for Loss experiences than for the other ACEs dimensions. Baseline CRP levels mediated respectively 
Table 2

Longitudinal mediation pathways: Direct associations between ACEs (total score and dimensions), CRP, and Depressive Symptoms.

\begin{tabular}{|c|c|c|c|c|c|c|c|c|c|c|c|c|c|c|c|c|}
\hline & \multicolumn{4}{|c|}{ CRP intercept } & \multicolumn{4}{|c|}{ CRP slope } & \multicolumn{4}{|c|}{ Depression intercept } & \multicolumn{4}{|c|}{ Depression slope } \\
\hline & $\beta$ & $\mathrm{SE}$ & $95 \% \mathrm{CI}$ & E-value & $\beta$ & $\mathrm{SE}$ & $95 \% \mathrm{CI}$ & E-value & $\beta$ & SE & $95 \% \mathrm{CI}$ & E-value & $\beta$ & SE & $95 \% \mathrm{CI}$ & E-value \\
\hline \multicolumn{17}{|l|}{ Model A } \\
\hline ACEs total score & 0.066 & 0.018 & $0.030 ; 0.102$ & $1.23(1.15)$ & 0.205 & 0.056 & $0.095 ; 0.315$ & $1.37(1.33)$ & 0.149 & 0.017 & $0.115 ; 0.183$ & $1.37(1.31)$ & 0.355 & 0.087 & $0.184 ; 0.526$ & $1.42(1.28)$ \\
\hline CRP Intercept & & & & & & & & & 0.145 & 0.021 & $0.104 ; 0.186$ & $1.36(1.29)$ & 0.215 & 0.046 & $0.124 ; 0.306$ & $1.30(1.22)$ \\
\hline CRP Slope & & & & & & & & & 0.339 & 0.154 & $0.037 ; 0.641$ & $1.66(1.16)$ & -0.111 & 0.380 & $-0.855 ; 0.633$ & - \\
\hline \multicolumn{17}{|l|}{ Model B } \\
\hline Threat & 0.034 & 0.019 & $-0.003 ; 0.071$ & - & 0.113 & 0.065 & $-0.014 ; 0.240$ & - & 0.035 & 0.047 & $-0.056 ; 0.126$ & - & 0.149 & 0.042 & $0.066 ; 0.232$ & 1.24 \\
\hline CRP Intercept & & & & & & & & & 0.155 & 0.021 & $0.114 ; 0.196$ & $1.38(1.31)$ & 0.238 & 0.046 & $0.147 ; 0.329$ & $1.32(1.24)$ \\
\hline CRP Slope & & & & & & & & & 0.366 & 0.216 & $-0.057 ; 0.789$ & - & -0.132 & 0.411 & $-0.937 ; 0.673$ & - \\
\hline \multicolumn{17}{|l|}{ Model C } \\
\hline & 0.049 & 0.016 & $0.017 ; 0.081$ & $1.20(1.11)$ & 0.155 & 0.052 & $0.054 ; 0.256$ & $1.30(1.16)$ & 0.031 & 0.017 & $-0.003 ; 0.065$ & - & 0.088 & 0.041 & $0.008 ; 0.168$ & $1.18(1.05)$ \\
\hline CRP Intercept & & & & & & & & & 0.155 & 0.021 & $0.114 ; 0.196$ & $1.38(1.31)$ & 0.243 & 0.047 & $0.150 ; 0.336$ & $1.33(1.24)$ \\
\hline CRP Slope & & & & & & & & & 0.357 & 0.159 & $0.046 ; 0.668$ & $1.69(1.18)$ & -0.143 & 0.441 & $-1.008 ; 0.722$ & - \\
\hline \multicolumn{17}{|l|}{ Model D } \\
\hline Household Dysfunction & 0.038 & 0.017 & $0.005 ; 0.071$ & $1.16(1.05)$ & 0.112 & 0.059 & $-0.004 ; 0.228$ & - & 0.110 & 0.019 & $0.073 ; 0.147$ & $1.31(1.24)$ & 0.199 & 0.044 & $0.113 ; 0.285$ & $1.29(1.20)$ \\
\hline CRP Intercept & & & & & & & & & 0.153 & 0.021 & $0.112 ; 0.194$ & $1.38(1.31)$ & 0.237 & 0.046 & $0.147 ; 0.327$ & $1.32(1.24)$ \\
\hline CRP Slope & & & & & & & & & 0.372 & 0.223 & $-0.065 ; 0.809$ & - & -0.111 & 0.397 & $-0.888 ; 0.666$ & - \\
\hline \multicolumn{17}{|l|}{ Model E } \\
\hline Low Parental Bonding & 0.036 & 0.019 & $0.000 ; 0.072$ & - & 0.119 & 0.060 & $0.002 ; 0.236$ & $1.26(1.02)$ & 0.125 & 0.019 & $0.088 ; 0.162$ & $1.33(1.27)$ & 0.250 & 0.042 & $0.168 ; 0.332$ & $1.34(1.26)$ \\
\hline CRP Intercept & & & & & & & & & 0.152 & 0.021 & $0.111 ; 0.193$ & $1.38(1.30)$ & 0.230 & 0.048 & $0.135 ; 0.325$ & $1.32(1.23)$ \\
\hline CRP Slope & & & & & & & & & 0.353 & 0.222 & $-0.081 ; 0.787$ & - & -0.127 & 0.399 & $-0.909 ; 0.655$ & - \\
\hline
\end{tabular}

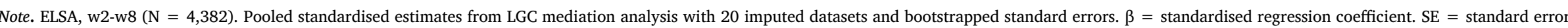

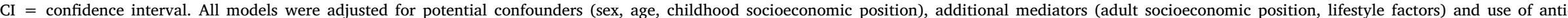
inflammatory/anti-hypertensive drugs (Model 1). $\beta$ coefficients in bold are statistically significant at the $95 \%$ confidence level. E-values are reported only for statistically significant associations. 


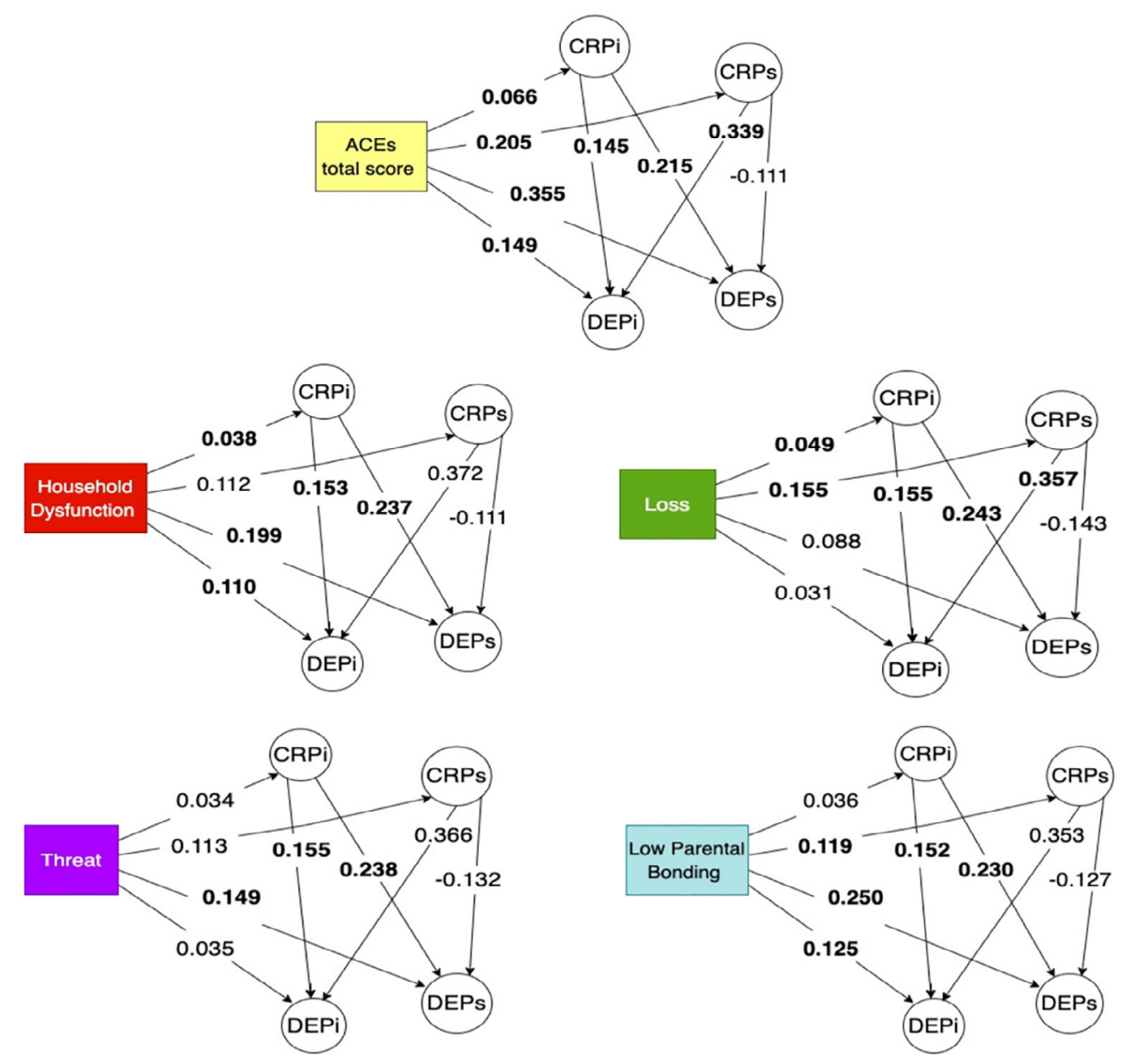

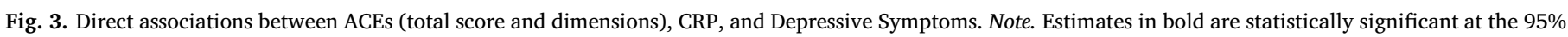

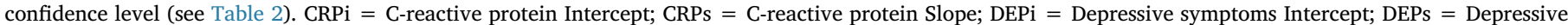

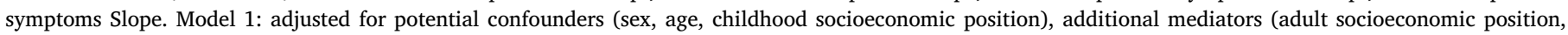
lifestyle factors) and use of anti-inflammatory/anti-hypertensive drugs. Standard errors and confidence intervals can be found in Table 2.

$20 \%$ and $12 \%$ of the remaining association of Loss with the baseline value and change in depressive symptoms, after other covariates had been taken into account. Sensitivity analyses showed that adult socioeconomic position and lifestyle factors had little influence on the mediation effects of CRP. The E-values suggested that unmeasured confounders showing relatively modest associations (risk ratio $=1.16$ or above) with ACEs, CRP, and depressive symptoms could explain away these relationships. However, the analysis did adjust for the most likely confounders and additional mediators, including childhood and adult socioeconomic position, lifestyle factors such as smoking and alcohol consumption, adiposity, and medications.

Numerous studies have investigated the relationship of inflammation with ACEs or depression (Baumeister et al., 2016; Iob et al., 2019; Haapakoski et al., 2015). However, very limited research has assessed these associations amongst older adults and formally tested the possible mediation role of inflammation with longitudinal data. Our results suggest that the relationship of ACEs with elevated CRP levels and depressive symptoms is likely to persist into later life, and that ACEs might also be related to a greater increase in these outcomes over time. These findings are consistent with the notion that inflammation might be an important psychobiological mechanism underlying the relationship of ACEs with depression. Hence, exposure to severe and chronic stress during early life might adversely affect the development of the immune system, resulting in chronically elevated and hyperactive inflammatory responses. This in turn may impair the development and function of the brain thereby increasing the individual's vulnerability to depression (Danese and Baldwin, 2017). One study recently found that greater exposure to psychosocial stress was related to the onset of depressive symptoms in a sample of adolescents partly via increasing levels of interleukins but not CRP (Flouri et al., 2020). The fact that CRP partially mediated the association between ACEs and depressive symptoms in this study of older adults could indicate that CRP is a more relevant biomarker of ACEs and depression in later life than at younger ages. Accordingly, the inflammatory contribution to depression seems to be particularly relevant to older adults since advancing age is linked to upregulation of the inflammatory response and greater risk of physical health comorbidities (Gallagher et al., 2017). In addition, recent evidence indicates that the relationship of ACEs with CRP is stronger in adults compared with children and adolescents (Kuhlman et al., 2019), possibly due to the presence of a hyporesponsive inflammatory response during early life (Simon et al., 2015).

In line with previous evidence, the average levels of depressive symptoms in the sample increased over time. But, unexpectedly, CRP levels showed a slight decrease across the timepoints. This result could be explained by the fact that ELSA is a population-based study of predominantly healthy older adults, with a relatively low prevalence of chronic physical health conditions linked to inflammation. Declines in CRP levels over time have in fact been documented also in other population-based studies of older adults (Stevenson, 2018). Hence, the small decline in CRP levels presented here could indicate a more successful ageing process in our sample.

It is also not clear whether different dimensions of ACEs might have 
Table 3

Longitudinal mediation pathways: Indirect associations of ACEs with Depressive Symptoms through CRP.

\begin{tabular}{|c|c|c|c|c|c|c|c|c|}
\hline \multirow[b]{2}{*}{ Mediation Pathway } & \multicolumn{4}{|c|}{ Model 1 (Adjusted for confounders and additional mediators) } & \multicolumn{4}{|c|}{ Model 2 (Confounders only) } \\
\hline & Indirect effect & SE & $95 \% \mathrm{CI}$ & Total effect mediated $(\%)^{\mathrm{a}}$ & Indirect effect & SE & $95 \% \mathrm{CI}$ & Total effect mediated $(\%)^{\mathrm{a}}$ \\
\hline \multicolumn{9}{|l|}{ ACEs total score } \\
\hline ACE-CRPi-DEPi & 0.006 & 0.002 & $0.002 ; 0.010$ & $7 \%$ & 0.006 & 0.002 & $0.002 ; 0.010$ & $6 \%$ \\
\hline ACE-CRPi-DEPs & 0.003 & 0.001 & $0.001 ; 0.005$ & $5 \%$ & 0.003 & 0.001 & $0.001 ; 0.005$ & $5 \%$ \\
\hline ACE-CRPs-DEPi & 0.041 & 0.023 & $-0.004 ; 0.086$ & - & 0.044 & 0.035 & $-0.025 ; 0.113$ & - \\
\hline ACE-CRPs-DEPs & -0.004 & 0.014 & $-0.031 ; 0.023$ & - & -0.005 & 0.009 & $-0.023 ; 0.013$ & - \\
\hline \multicolumn{9}{|l|}{ Threat } \\
\hline Threat-CRPi-DEPi & 0.014 & 0.008 & $-0.002 ; 0.030$ & - & 0.014 & 0.008 & $-0.002 ; 0.030$ & - \\
\hline Threat-CRPi-DEPs & 0.007 & 0.004 & $-0.001 ; 0.015$ & - & 0.007 & 0.004 & $-0.001 ; 0.015$ & - \\
\hline Threat-CRPs-DEPi & 0.118 & 0.116 & $-0.109 ; 0.345$ & - & 0.118 & 0.13 & $-0.137 ; 0.373$ & - \\
\hline Threat-CRPs-DEPs & -0.012 & 0.041 & $-0.092 ; 0.068$ & - & -0.014 & 0.028 & $-0.069 ; 0.041$ & - \\
\hline \multicolumn{9}{|l|}{ Loss } \\
\hline Loss-CRPi-DEPi & 0.016 & 0.006 & $0.004 ; 0.028$ & $20 \%$ & 0.016 & 0.006 & $0.004 ; 0.028$ & $15 \%$ \\
\hline Loss -CRPi-DEPs & 0.007 & 0.003 & $0.001 ; 0.013$ & $12 \%$ & 0.007 & 0.003 & $0.001 ; 0.013$ & $8 \%$ \\
\hline Loss-CRPs-DEPi & 0.117 & 0.07 & $-0.020 ; 0.254$ & - & 0.13 & 0.106 & $-0.078 ; 0.338$ & - \\
\hline Loss -CRPs-DEPs & -0.012 & 0.039 & $-0.088 ; 0.064$ & - & -0.015 & 0.025 & $-0.064 ; 0.034$ & - \\
\hline \multicolumn{9}{|l|}{ Household dysfunction } \\
\hline HouseDys-CRPi-DEPi & 0.011 & 0.005 & $0.001 ; 0.021$ & $5 \%$ & 0.011 & 0.005 & $0.001 ; 0.021$ & $5 \%$ \\
\hline HouseDys-CRPi-DEPs & 0.005 & 0.003 & $-0.001 ; 0.011$ & - & 0.005 & 0.003 & $-0.001 ; 0.011$ & - \\
\hline HouseDys-CRPs-DEPi & 0.079 & 0.069 & $-0.056 ; 0.214$ & - & 0.075 & 0.069 & $-0.060 ; 0.210$ & - \\
\hline HouseDys-CRPs-DEPs & -0.006 & 0.026 & $-0.057 ; 0.045$ & - & -0.008 & 0.017 & $-0.041 ; 0.025$ & - \\
\hline \multicolumn{9}{|l|}{ Low parental bonding } \\
\hline Bonding-CRPi-DEPi & 0.010 & 0.005 & $0.000 ; 0.020$ & - & 0.010 & 0.005 & $0.000 ; 0.020$ & - \\
\hline Bonding-CRPi-DEPs & 0.005 & 0.003 & $-0.001 ; 0.011$ & - & 0.005 & 0.003 & $-0.001 ; 0.011$ & - \\
\hline Bonding-CRPs-DEPi & 0.077 & 0.071 & $-0.062 ; 0.216$ & - & 0.081 & 0.072 & $-0.060 ; 0.222$ & - \\
\hline Bonding-CRPs-DEPs & 0.007 & 0.026 & $-0.058 ; 0.044$ & - & -0.009 & 0.016 & $-0.040 ; 0.022$ & - \\
\hline
\end{tabular}

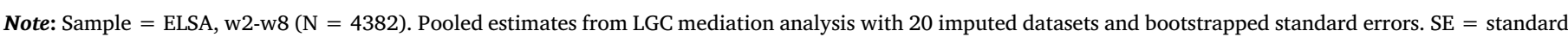

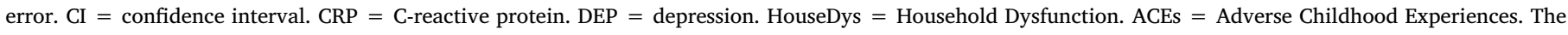

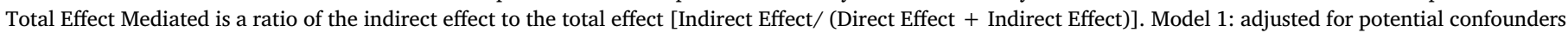

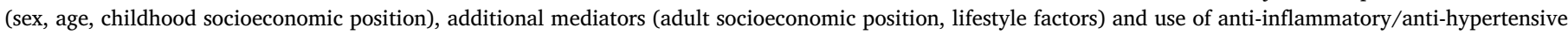

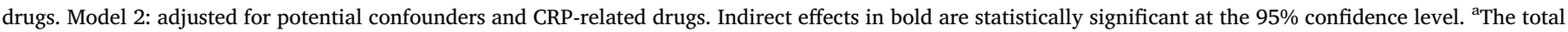
effect mediated is reported only for statistically significant indirect effects.

distinct psychological and physiological effects on child development. In our study the dimension representing experiences related to loss of an attachment figure had stronger associations with CRP, which in turn explained a larger proportion of its association with depressive symptoms, compared with the effects of other ACEs dimensions. However, the differences between the indirect associations of Loss and those of the other ACEs dimensions were small. These results are in contrast with previous studies using the Threat-Deprivation model (McLaughlin et al., 2014), which showed that ACEs involving harm or threat of harm have stronger relationships with stress-related biological outcomes (e.g. cortisol) than ACEs related to deprivation (Busso et al., 2017; Sumner et al., 2019; LoPilato, 2019). However, the Threat-Deprivation model is not well suited to represent the ACEs dimensions found in our study. Notably, it does not capture certain adversities (e.g. parental substance abuse and separation), and some deprivation experiences are likely to belong to distinct sub-dimensions (e.g. parental loss and neglect) (Iob et al., 2019). On the other hand, the results regarding Loss experiences are in line with the findings of earlier analyses suggesting that CRP might be predominantly related to parental absence during childhood, whereas its associations with other types of adversities seem to be weaker (Baumeister et al., 2016). In addition, they are partially consistent with the Biological Salience model which highlights the likely adverse biological consequences of disruptions in the caregiving environment (e.g. death of a parent, parental separation) for the development of the central nervous system and downstream neuroendocrine and inflammatory responses linked to mental and physical health (Kuhlman et al., 2017). Nevertheless, it is also important to note that most dimensions of ACEs were related to higher CRP and depressive symptoms, and their cumulative effect exhibited the strongest relationship with these outcomes. Consequently, all types of ACEs assessed in this study are likely to be important risk factors for elevated inflammation and depressive symptoms in later life.

Our study has several important strengths. We used a large population-based sample of older adults. Participants were not selected on the basis of exposure to ACEs or presence of depressive symptoms and were therefore more representative of the general population. We were also able to assess the mediation effects of CRP with greater subtlety owing to the availability of repeated measures of CRP and depressive symptoms. Another strength of our analysis concerns the measurement of different dimensions of ACEs in addition to the use of a cumulative risk score, which enabled us to assess the specific associations of distinct ACEs dimensions separately. Despite this, a number of limitations should be noted. First, as suggested by the E-values, our results could be prone to unmeasured confounding bias. Although the analysis was adjusted for relevant confounders and additional mediators, it is necessary to consider other potential sources of unmeasured confounding associated with our exposure, mediator, and outcome variables when interpreting the results (Blum et al., 2020). These might include, for instance, different childhood socioeconomic characteristics than those included in our study (e.g. area deprivation, parental social networks) (Allen and Donkin, 2015) or genetically influenced individual traits and vulnerabilities (Dalvie, 2020; Schoeler, 2019). Second, ACEs were assessed retrospectively based on the participants' recollections of their childhood experiences. Such measures are particularly prone to measurement error arising from the participants' motivations, personality styles, cognitive function, and memory biases, particularly at older ages (Hardt and Rutter, 2004). Biases in disclosing childhood experiences could also account for the high numbers of missing data in certain ACEs measures (Table 1). A recent meta-analysis has demonstrated that agreement between prospective and retrospective ACEs measures for 
experiences such as abuse and neglect is substantially lower than for other more clear-cut types of ACEs like parental loss (Baldwin et al., 2019). This could explain why Loss experiences exhibited larger associations with the outcomes than the other ACEs dimensions in this sample of older adults. It is also important to note that, despite the large number of studies on ACEs, a concrete and agreed-upon definition of this construct has not been achieved yet. There is a lack of consistency in the way in which ACEs are defined and measured across different studies (Lacey and Minnis, 2020). Consequently, our results cannot be directly compared with those of studies including different ACE items. Third, the mediation effects of CRP were generally small. This suggests that other markers of inflammation or different biological mechanisms might also play a role in the association between ACEs and depression. For instance, different lines of research suggest that neuroendocrine responses, microbiome alterations, neurocognitive processes, and epigenetic changes are all likely to be involved in the link between ACEs and mental health, as well as being associated with inflammation (Berens et al., 2017). Hence, it would be important to understand how these different biological processes influence each other in the aetiology of depression. Furthermore, several studies have found that the association between inflammation and depressive symptoms might be predominantly driven by the somatic symptoms of depression, whereas associations with cognitive-affective symptoms tend to be much smaller (White et al., 2017; Jokela et al., 2016; Chu, 2019; Iob et al., 2019). Along similar lines, a recent analysis found that CRP mediated the relationship of polygenic susceptibility to depression with somatic depressive symptoms, but not that with cognitive-affective symptoms (Frank et al., 2020). The mediation effects of inflammation found in our study could therefore be more strongly related to somatic symptoms of depression, but further work is needed to assess this possibility. Fourth, a considerable number of ELSA participants were not included in the study since they did not have data on at least one measure of ACEs, CRP, and depressive symptoms. Sensitivity analyses demonstrated that our analytical sample had better socioeconomic, health, and lifestyle characteristics than the full ELSA sample, although such differences were small. This could pose some limitations to the representativeness of the sample. Lastly, the trajectories of CRP and depressive symptoms were examined over a relatively short period which may not be sufficient to provide an accurate representation of their change during later life.

More prospective studies are needed to investigate the mediation role of inflammation and other stress-related biological processes in different samples of older adults and young people. Future studies should also consider the potentially different physiological effects of different types of ACEs and take into account a wider range of potential confounders if possible, including both individual and contextual-level socioeconomic characteristics and genetic factors. Furthermore, it would be important to determine whether there might be sensitive periods during early development when environmental exposures could have more powerful and enduring consequences for the development of the immune system and the brain.

\section{Conclusions}

To conclude, greater cumulative exposure to ACEs was associated not only with elevated CRP and depressive symptoms at baseline but also with their increase over time in a large population-based sample of older adults, independently of possible confounders (sex, age, and childhood socioeconomic position) and additional mediators (adult socioeconomic position and lifestyle factors). ACEs were related to depressive symptoms partly via higher CRP levels. CRP had larger mediation effects in the pathways linking Loss experiences with depression than in those involving other ACEs dimensions. These results suggest that inflammation might be one of the psychobiological mechanisms underlying the relationship of ACEs with depression. However, the mediation effects of CRP were generally small and susceptible to possible unmeasured confounding. Other inflammatory markers and different stress-related biological processes should also be considered in order to provide a more comprehensive understanding of the biological embedding of ACEs. Taken together, our findings highlight the importance of adopting an ACE-informed approach in the prevention and treatment of depression. Psychosocial and behavioural interventions to prevent ACEs and reduce their biological impact on children's development might help to lower the risk of elevated inflammation and depression in the population, as well as of other medical conditions linked with these disorders.

\section{Declaration of Competing Interest}

The authors declare that they have no known competing financial interests or personal relationships that could have appeared to influence the work reported in this paper.

\section{Acknowledgments}

Funding: Eleonora Iob is funded by the ESRC-BBSRC Soc-B Centre for Doctoral Training (ES/P000347/1). Rebecca Lacey receives funding from the ESRC (ES/P010229/1). The English Longitudinal Study of Ageing was developed by a team of researchers based at University College London, NatCen Social Research, the Institute for Fiscal Studies, and the University of Manchester. The data were collected by NatCen Social Research. The funding is provided by the National Institute of Aging (R01AG017644) and a consortium of UK government departments coordinated by the Economic and Social Research Council. The developers and funders of the English Longitudinal Study of Ageing and the UK Data Archive do not bear any responsibility for the analyses or interpretations presented here.

\section{Contributors}

All authors contributed significantly to the conception, design, analysis or interpretation of data and were involved in revising it critically for intellectual context. The final submission of this paper was approved by all authors.

\section{Appendix A. Supplementary data}

Supplementary data to this article can be found online at https:// doi.org/10.1016/j.bbi.2020.07.045.

\section{References}

Patel, V., et al., 2016. Addressing the burden of mental, neurological, and substance use disorders: key messages from Disease Control Priorities. Lancet 387, 1672-1685.

James, S.L., et al., 2018. Global, regional, and national incidence, prevalence, and years lived with disability for 354 diseases and injuries for 195 countries and territories, 1990-2017: a systematic analysis for the Global Burden of Disease Study 2017. Lancet 392, 1789-1858.

Kivimäki, M., Batty, G. D., Steptoe, A., Kawachi, I. \& Steptoe, A. Depression and negative emotions. in The Routledge International Handbook of Psychosocial Epidemiology 136-155 (Routledge, 2019).

Global Burden of Disease Collaborative Network. Global Burden of Disease Study 2017 (GBD 2017) Results. (2018).

Kok, R.M., Reynolds, C.F., 2017. Management of depression in older adults. JAMA 317, 2114.

Royal College of Psychiatrists. Suffering in silence: age inequality in older people's mental health care. (2018).

Tampubolon, G., Maharani, A., 2017. When did old age stop being depressing? Depression trajectories of older americans and britons 2002-2012. Am. J. Geriatr. Psychiatry 25, 1187-1195.

Fiske, A., Wetherell, J.L., Gatz, M., 2009. Depression in older adults. Annu. Rev. Clin. Psychol. 5, 363-389.

Rodda, J., Walker, Z. \& Carter, J. Depression in older adults. BMJ 343, d5219-d5219 (2011).

McLaughlin, K.A., 2016. Future directions in childhood adversity and youth psychopathology. J. Clin. Child Adolesc. Psychol. 45, 361-382.

Felitti, V.J., et al., 1998. Relationship of childhood abuse and household dysfunction to 
many of the leading causes of death in adults. Am. J. Prev. Med. 14, 245-258.

Dong, M., et al., 2004. The interrelatedness of multiple forms of childhood abuse, neglect, and household dysfunction. Child Abuse Negl. 28, 771-784.

Finkelhor, D., Shattuck, A., Turner, H., Hamby, S., 2015. A revised inventory of adverse childhood experiences. Child Abus. Negl. 48, 13-21.

Finkelhor, D., Shattuck, A., Turner, H., Hamby, S., 2013. Improving the adverse childhood experiences study scale. Arch. Pediatr. Adolesc. Med. 167, 70-75.

Hughes, K., et al., 2017. The effect of multiple adverse childhood experiences on health: a systematic review and meta-analysis. Lancet Public Heal. 2, e356-e366.

Houtepen, L.C., Heron, J., Suderman, M.J., Tilling, K., Howe, L.D., 2018. Adverse childhood experiences in the children of the avon longitudinal study of parents and children (ALSPAC). Wellcome Open Res. 3, 106.

Bellis, M.A., Hughes, K., Leckenby, N., Perkins, C., Lowey, H., 2014. National household survey of adverse childhood experiences and their relationship with resilience to health-harming behaviors in England. BMC Med. 12.

Merrick, M.T., Ford, D.C., Ports, K.A., Guinn, A.S., 2018. Prevalence of adverse childhood experiences from the 2011-2014 behavioral risk factor surveillance system in 23 states. JAMA Pediatr. 172, 1038.

Bellis, M.A., et al., 2019. Articles life course health consequences and associated annual costs of adverse childhood experiences across Europe and North America: a systematic review and meta-analysis. Lancet Public Heal. 4, e517-e528.

Nanni, V., Uher, R., Danese, A., 2012. Childhood maltreatment predicts unfavorable course of illness and treatment outcome in depression: a meta-analysis. Am. J. Psychiatry 169, 141-151.

Cheong, E.V., Sinnott, C., Dahly, D., Kearney, P.M., 2017. Adverse childhood experiences (ACEs) and later-life depression: perceived social support as a potential protective factor. BMJ Open 7, 1-11.

Danese, A., Baldwin, J.R., 2017. Hidden Wounds? Inflammatory links between childhood trauma and psychopathology. Annu. Rev. Psychol. 68, 517-544.

Baumeister, D., Akhtar, R., Ciufolini, S., Pariante, C.M., Mondelli, V., 2016. Childhood trauma and adulthood inflammation: a meta-analysis of peripheral C-reactive protein, interleukin- 6 and tumour necrosis factor- $\alpha$. Mol. Psychiatry 21, 642-649.

Iob, E., Lacey, R., Steptoe, A., 2019. The long-term association of adverse childhood experiences with C-reactive protein and hair cortisol: cumulative risk versus dimensions of adversity. Brain. Behav. Immun. https://doi.org/10.1016/j.bbi.2019.12. 019.

Miller, A., Maletic, V., Raison, C., 2009. Inflammation and Its discontents: the role of cytokines in the pathophysiology of major depression. Biol. Psychiatry 65, 732-741.

Haapakoski, R., Mathieu, J., Ebmeier, K.P., Alenius, H., Kivimäki, M., 2015. Cumulative meta-analysis of interleukins 6 and $1 \beta$, tumour necrosis factor $\alpha$ and C-reactive protein in patients with major depressive disorder. Brain. Behav. Immun. 49, 206-215.

Enache, D., Pariante, C.M., Mondelli, V., 2019. Markers of central inflammation in major depressive disorder: a systematic review and meta-analysis of studies examining cerebrospinal fluid, positron emission tomography and post-mortem brain tissue. Brain Behav. Immun. 81, 24-40.

Flouri, E., Francesconi, M., Midouhas, E., Lewis, G., 2020. Prenatal and childhood adverse life events, inflammation and depressive symptoms across adolescence. J. Affect. Disord. 260, 577-582.

Tang, Y., Fung, E., Xu, A., Lan, H.-Y., 2017. C-reactive protein and ageing. Clin. Exp. Pharmacol. Physiol. 44, 9-14.

Kuhlman, K.R., Horn, S.R., Chiang, J.J., Bower, J.E., 2019. Early life adversity exposure and circulating markers of inflammation in children and adolescents: a systematic review and meta-analysis. Brain. Behav. Immun. https://doi.org/10.1016/j.bbi.2019. 04.028.

McLaughlin, K.A., Sheridan, M.A., 2016. Beyond cumulative risk: a dimensional approach to childhood adversity. Curr. Dir. Psychol. Sci. 25, 239-245.

Busso, D.S., McLaughlin, K.A., Sheridan, M.A., 2017. Dimensions of adversity, physiological reactivity, and externalizing psychopathology in adolescence: deprivation and threat. Psychosom. Med. 79, 162-171.

Sumner, J.A., Colich, N.L., Uddin, M., Armstrong, D., McLaughlin, K.A., 2019. Early experiences of threat, but not deprivation, are associated with accelerated biological aging in children and adolescents. Biol. Psychiatry 85, 268-278.

Lacey, R.E., Pinto Pereira, S.M., Li, L., Danese, A., 2020. Adverse childhood experiences and adult inflammation: single adversity, cumulative risk and latent class approaches. Brain. Behav. Immun. https://doi.org/10.1016/j.bbi.2020.03.017.

Zaninotto, P. \& Steptoe, A. English Longitudinal Study of Ageing. in Encyclopedia of Gerontology and Population Aging 1-7 (Springer International Publishing, 2019). doi:10.1007/978-3-319-69892-2_335-1.

Steptoe, A., Breeze, E., Banks, J., Nazroo, J., 2013. Cohort profile: the english longitudinal study of ageing. Int. J. Epidemiol. 42, 1640-1648.

Pearson, T.A., et al., 2003. Markers of inflammation and cardiovascular disease. Circulation 107, 499-511.

Parker, G., Tupling, H., Brown, L.B., 1979. A parental bonding instrument. Br. J. Med. Psychol. 52, 1-10.

Taylor-Robinson, D.C., Straatmann, V.S., Whitehead, M., 2018. Adverse childhood experiences or adverse childhood socioeconomic conditions? Lancet Public Heal. 3, e262-e263.

Amso, D., Lynn, A., 2017. Distinctive mechanisms of adversity and socioeconomic inequality in child development: a review and recommendations for evidence-based policy. Policy Insights Behav. Brain Sci. 4, 139-146.

Walsh, D., McCartney, G., Smith, M., Armour, G., 2019. Relationship between childhood socioeconomic position and adverse childhood experiences (ACEs): a systematic review. J. Epidemiol. Commun. Health 73, 1087-1093.

42. Ward, K., Medina, J., Mo, M. \& Cox, K. ELSA Wave Three: Life History Interview A user guide to the data. (2009).

NatCen Social Research. English Longitudinal Study of Ageing (ELSA): User Guide to the Nurse Visit Datasets. Waves 2, 4, 6, 8. (2018).

Graig, R., Deverill, C. \& Pickering, K. Quality control of blood, saliva and urine analyses. in Health survey for England 2004, Methodology and documentation Vol. 2 (eds. Spronston, K. \& Mindell, J.) 34-41 (2006).

Radloff, L.S., 1977. The CES-D scale: a self-report depression scale for research in the general population. Appl. Psychol. Meas. 1, 385-401.

White, J., et al., 2016. Duration of depressive symptoms and mortality risk: the English Longitudinal study of ageing (ELSA). Br. J. Psychiatry 208, 337-342.

Zivin, K., et al., 2010. Depression among older adults in the United States and England. Am. J. Geriatr. Psychiatry 18, 1036-1044.

Karim, J., Weisz, R., Bibi, Z., ur Rehman, S., 2015. Validation of the Eight-Item Center for Epidemiologic Studies Depression Scale (CES-D) Among Older Adults. Curr. Psychol. 34, 681-692.

Andresen, E.M., Malmgren, J.A., Carter, W.B., Patrick, D.L., 1994. Screening for depression in well older adults: evaluation of a short form of the CES-D. Am. J. Prev. Med. $10,77-84$.

Turvey, C.L., Wallace, R.B., Herzog, R., 1999. A revised CES-D measure of depressive symptoms and a DSM-based measure of major depressive episodes in the elderly. Int Psychogeriatr. 11, 139-148.

Schisterman, E.F., Cole, S.R., Platt, R.W., 2009. Overadjustment bias and unnecessary adjustment in epidemiologic studies. Epidemiology 20, 488-495.

Selig, J.P., Preacher, K.J., 2009. Mediation models for longitudinal data in developmental research. Res. Hum. Dev. 6, 144-164.

MacKinnon, D.P., Fairchild, A.J., Fritz, M.S., 2007. Mediation analysis. Annu. Rev. Psychol. 58, 593-614.

Hayes, A.F., 2009. Beyond Baron and Kenny: Statistical mediation analysis in the new millennium. Commun. Monogr. 76, 408-420.

VanderWeele, T.J., Ding, P., 2017. Sensitivity analysis in observational research: introducing the E-Value. Ann. Int. Med. 167, 268-274.

Rubin, D.B., 2004. Multiple Imputation for Nonresponse in Surveys. John Wiley and Sons.

Blum, M.R., Tan, Y.J., Ioannidis, J.P.A., 2020. Use of E-values for addressing confounding in observational studies-an empirical assessment of the literature. Int. J. Epidemiol. https://doi.org/10.1093/ije/dyz261.

Gallagher, D., Kiss, A., Lanctot, K., Herrmann, N., 2017. Depression with inflammation: longitudinal analysis of a proposed depressive subtype in community dwelling older adults. Int. J. Geriatr. Psychiatry 32, e18-e24.

Simon, A.K., Hollander, G.A., McMichael, A., 2015. Evolution of the immune system in humans from infancy to old age. Proc. R. Soc. B Biol. Sci. 282.

Stevenson, A.J., et al., 2018. Trajectories of inflammatory biomarkers over the eighth decade and their associations with immune cell profiles and epigenetic ageing. Clin. Epigenet. 10, 159.

McLaughlin, K.A., Sheridan, M.A., Lambert, H.K., 2014. Childhood adversity and neural development: deprivation and threat as distinct dimensions of early experience. Neurosci. Biobehav. Rev. 47, 578-591.

LoPilato, A.M., et al., 2019. Stress perception following childhood adversity: unique associations with adversity type and sex. Dev. Psychopathol. 1-14. https://doi.org/10. 1017/S0954579419000130.

Kuhlman, K.R., Chiang, J.J., Horn, S., Bower, J.E., 2017. Developmental psychoneuroendocrine and psychoneuroimmune pathways from childhood adversity to disease. Neurosci. Biobehav. Rev. 80, 166-184.

Allen, M. \& Donkin, A. The impact of adverse experiences in the home on the health of children and young people, and inequalities in prevalence and effects. (UCL Institute of Health Equity, 2015).

Dalvie, S., et al., 2020. Genomic influences on self-reported childhood maltreatment. Transl. Psychiatry 10, 1-12.

Schoeler, T., et al., 2019. Multi-polygenic score approach to identifying individual vulnerabilities associated with the risk of exposure to bullying. JAMA Psychiatry 76, 730-738.

Hardt, J., Rutter, M., 2004. Validity of adult retrospective reports of adverse childhood experiences: review of the evidence. J. Child Psychol. Psychiatry Allied Discip. 45, $260-273$.

Baldwin, J.R., Reuben, A., Newbury, J.B., Danese, A., 2019. Agreement between prospective and retrospective measures of childhood maltreatment. JAMA Psychiatry 76, 584.

Lacey, R.E., Minnis, H., 2020. Practitioner review: twenty years of research with adverse childhood experience scores - Advantages, disadvantages and applications to practice. J. Child Psychol. Psychiatry 61, 116-130.

Berens, A.E., Jensen, S.K.G., Nelson, C.A., 2017. Biological embedding of childhood adversity: from physiological mechanisms to clinical implications. BMC Med. 15, 135.

White, J., Kivimäki, M., Jokela, M., Batty, G.D., 2017. Association of inflammation with specific symptoms of depression in a general population of older people: the english longitudinal study of ageing. Brain Behav. Immun. 61, 27-30.

Jokela, M., Virtanen, M., Batty, G.D., Kivimäki, M., 2016. Inflammation and specific symptoms of depression. JAMA Psychiatry 73, 87.

Chu, A.L., et al., 2019. Longitudinal association between inflammatory markers and specific symptoms of depression in a prospective birth cohort. Brain. Behav. Immun. 76, 74-81.

Iob, E., Kirschbaum, C., Steptoe, A., 2019. Persistent depressive symptoms, HPA-axis hyperactivity, and inflammation: the role of cognitive-affective and somatic symptoms. Mol. Psychiatry 1-11. https://doi.org/10.1038/s41380-019-0501-6.

Frank, P., Ajnakina, O., Steptoe, A., Cadar, D., 2020. Genetic susceptibility, inflammation and specific types of depressive symptoms: evidence from the English Longitudinal Study of Ageing. Transl. Psychiatry 10, 1-9. 